\title{
Shear-Induced Amyloid Formation in the Brain: II. An Experimental System for Monitoring Amyloid Shear Processes and Investigating Potential Spinal Tap Problems
}

\author{
Conrad N. Trumbore* \\ Department of Chemistry and Biochemistry, University of Delaware, Kennett Square, PA, USA
}

Accepted 16 May 2017

\begin{abstract}
Liquid sheared amyloid- $\beta(A \beta)$ initiates amyloid cascade reactions, producing unstable, potentially toxic oligomers. There is a need for new analytical tools with which to study these oligomers. A very small bore capillary flow system is proposed as a tool for studying the effects of liquid shear in amyloid research. This simple system consists of injecting a short cylindrical liquid sample plug containing dissolved amyloid into a liquid mobile phase flowing through an empty, very small internal diameter capillary tube. For liquid samples containing a single protein sample, under conditions in which there is laminar flow and limited sample protein molecular diffusion, chromatograms monitoring the optical protein absorbance of capillary effluent contain either one or two peaks, depending on the mobile phase flow rate. By controlling the sample diffusion times through changes in flow rate and/or capillary diameter, this tool can be used to generate aliquot samples with precise, reproducible amounts of shear for exploring the effects of variable shear on amyloid systems. The tool can be used for producing in-capillary stopped flow spectra of shear-stressed $A \beta$ monomers as well as for kinetic studies of $A \beta$ dimer- and oligomer-forming reactions between shear stressed $A \beta$ monomers. Many other experiments are suggested using this experimental tool for studying the effects of shear on different $A \beta$ and other amyloid systems, including testing for potentially serious amyloid sampling errors in spinal tap quantitative analysis. The technique has potential as both a laboratory research and a clinical tool.
\end{abstract}

Keywords: Aggregation, amyloid- $\beta$, amyloid cascade, amyloid dimer, amyloid oligomers, capillary analysis, limited diffusion, protein misfolding, spinal tap

\section{INTRODUCTION}

In the first paper in this series [1], this author proposed that liquid shear processes taking place in different brain regions may be responsible for

\footnotetext{
${ }^{*}$ Correspondence to: Conrad N. Trumbore, Department of Chemistry and Biochemistry, University of Delaware, 441 Crosslands Drive, Kennett Square, PA 19348, USA. Tel.: +1 610388 7093; Fax: +1 610388 5691; E-mail: conradt@udel.edu.
}

initiating an amyloid chemical reaction cascade leading from amyloid- $\beta(A \beta)$ monomers to both solid cerebral amyloid angiopathy vascular deposits and neurotoxic $A \beta$ oligomers and ultimately to Alzheimer's disease (AD) plaques [2, 3]. Experimental evidence [4-6] demonstrates that relatively weak shear forces that are not able to break the numerous hydrogen bonds necessary to even partially unfold amyloid proteins are able to induce 
subtle protein conformational changes, especially in the group of intrinsically disordered proteins (IDPs) [7] that includes $A \beta$. Since experiments have shown that such gentle shear has induced the amyloid cascade reaction in $A \beta$ [4], and since recent experiments have also demonstrated that $\mathrm{A} \beta$-containing cerebral fluids such as cerebrospinal fluid (CSF) and interstitial fluid (ISF) are not quiescent in different parts of the rat brain $[8,9]$, it is suggested that shear may produce a variety of new high energy $A \beta$ molecular conformations in different brain regions, depending on the shear rates present in those regions [1].

The exact nature of the flow rates and flow patterns of both ISF and CSF is the subject of much research and debate [8-11]. Regardless of the exact structure of the flow paths or brain fluid flow rates found in the extracellular spaces (ECS) of the brain parenchyma, it is suggested [1] that exterior membrane surfaces of neurons and possibly glia could play critical roles in neuronal brain disease if shear is an important factor in generating amyloid-related toxicity. This is because the regions on and near these surfaces would be those brain regions in which there would be maximum liquid shear even if there is a relatively slow ISF flow past them. Such shear is proposed to lead to a membrane chemical reaction sequence that is suggested [1] as an alternate mechanism to one proposed for the formation of toxic membrane oligomers.

It is also suggested [1] that tortuous CSF and possibly ISF flow paths in the region within and around the walls of blood vessels leading into the brain are regions where liquid shear of $A \beta$ molecules in CSF probably causes the formation of solid deposits associated with cerebral amyloid angiopathy consisting mainly of $A \beta_{40}$. Such deposits have been suggested to be responsible for the slowing of the clearance of $A \beta$ from the brain parenchyma [12]. Increasing $\mathrm{A} \beta$ concentrations in CSF is an important biomarker for AD [13]. Even though the parenchymal ISF flow rates are apparently very low in comparison with flow rates along arterial CSF flow paths, there are much tighter constrictions of the parenchymal ISF flow path because of the high density of neurons, astrocytes, glia, and microglia [14]. ISF flow through these narrow ECS paths between and around these brain structures is additionally impeded by the components of perineuronal networks [15] either projecting out from the cell membranes anchors or suspended within this path. These ISF flow obstacles create tortuous flow paths that heavily restrict ISF flow rates. Such paths generate at least two types of shear, laminar and extensional, both of which are thought to be present in the brain [1]. Because of the greater shear sensitivity of $A \beta_{42}$, this isomer is trapped mainly within the parenchyma by shear-induced $A \beta$ aggregation processes.

It is suggested that under shear conditions, $A \beta$ is distorted into a higher energy, metastable conformation, designated $A \beta^{*}$. This excited molecule is somewhat like a highly flexible squeezed foam rubber ball in that when the shear is released on the molecule, the molecule returns to its native, unstressed conformation. (Supplement C should be consulted for justification of this model.) The time taken for the return to the unstressed conformation, called the $A \beta^{*}$ relaxation time, will vary, depending on both the shear rate (how much force is used to compress the ball?) and the time spent under stress (how quickly air returns when the ball is compressed and released?). $\mathrm{A} \beta *$ is a generic term that encompasses a whole range of distortion energies that depend on the liquid shear rate. The energy of $A \beta^{*}$ will be quite different in different parts of the brain. These various energy shear-induced intermediates may lead to different oligomer isoforms.

If, as outlined above, shear is indeed an important factor in initiating amyloid cascade reactions in the brain leading to toxic oligomers associated with membranes and precursors to amyloid plaque, a number of questions follow. What laboratory model or system can be used to simulate the kinds of shear encountered in the brain? Since it is very difficult to study experimentally the type of shear processes suggested above within the brain parenchyma and arterial system, how can researchers most effectively study in the laboratory the molecular effects of shear on amyloids in a brain-like environment?

\section{EXPERIMENTAL CHALLENGES IN STUDYING BRAIN LIQUID SHEAR}

One amyloid review [16] suggests: "A wealth of structural data exists for the relatively benign fibrils of $A \beta$; however, intermediate structures such as structured monomers and oligomers have remained relatively unexplored, largely due to their transient nature. In this regard, the inability to crystallize and/or trap a pure (single state) sample has presented much of the difficulty in performing structural studies of oligomeric species." Several other reviews have also similarly called for improvements in analytical techniques in amyloid research [17]. This implies that, in order to study early events in the shear initiated 
amyloid cascade reaction, measurements may need to be taken while shear stressing exists or immediately after the $A \beta$ is released from shear stress.

Opportunities for liquid shear processes within the brain are many and highly variable [1]. Not only are there numerous combinations of laminar and extensional shear possible, but there are also claims of oscillating CSF and ISF flow [10,11]. Such back-andforth flows would generate oscillating shear fields in proximity to large molecules found in the perineuronal network and basement membrane molecular components that impede both CSF and ISF flow.

Thus, there is one important complicating factor that needs to be incorporated into any shear discussion and experimental program involving shear. What are the opportunities, when there is little or no shear present, for shear-excited $A \beta^{*}$ molecules (Supplement $C$ ) to relax by losing their shear energy to their surroundings as thermal energy, accompanying a conformation change to a lower energy "ground state" $\mathrm{A} \beta$ conformation? In other words, what is the natural lifetime of a shear-excited $A \beta^{*}$ molecule in the absence of shear? Is this lifetime shorter or longer than the in vivo shear oscillation cycles involving ISF and CSF discussed above? This could be determined experimentally by suddenly stopping the shear flow and observing spectroscopically any conformation changes taking place in the stopped segment of the shear distorted cylindrical plug contained in the capillary detector after the moment of stoppage. See below for a more complete discussion of this experimental system.

Thus, variation in flow rates and oscillating changes in flow direction present many experimental challenges in simulating these complex types of shear situations. It would appear to this author that much more fundamental laboratory shear studies need to be undertaken to understand the role of different types of shear in amyloid disease. We previously studied only the simplest shear type, namely laminar shear in metal capillary tubing of proteins in general [18]. These now need to be repeated with many different amyloid compounds. Simple laminar shear next to the simple brain cell wall can be simulated by the surfaces of very small diameter, flow-restricting capillaries. Very closely-spaced, flat plates, whose inside walls, in some instances coated with a membranemimicking substance, could also be studied for ease of surface analysis after flow experiments. Obviously, the surface chemical composition of the two types of wall, membranes and metal capillary, are not the same. However, at the very minimum, the predomi- nant attractive force between dissolved shear-stressed proteins and the metal capillary surface is probably polar, and the inside surfaces of the capillary can be modified and coated with various chemicals to study more membrane-like surfaces and the effects of metals on the aggregation process. Leakage of metal ions into the fluid medium from the metal surface also needs to be considered since they may affect the $A \beta$ aggregation process.

Both sheared $A \beta$ molecules and membrane surfaces [19] will have attractive regions because of exposed hydrophobic, polar, and ionic forces. The higher the shear rate, the more stretched the $A \beta$ molecule will be, exposing and attracting certain regions to corresponding similar regions in the membrane, causing $\mathrm{A} \beta$-membrane adsorption. What kind of proteins are embedded within and projecting from the membrane will generate new extensional shear regions.

Our early research efforts have been with stainlesssteel capillaries with a $0.25 \mathrm{~mm}$ inner diameter [18]. It should be noted here that this inner diameter is very close, if not identical, to that of the needles used for spinal taps used to obtain CSF from AD patients for analysis of amyloids. This dimension is still orders of magnitude larger than the dimensions estimated [14] for those of the ECS within the brain parenchyma. This early research led us to suggest that the shear next to the capillary wall led to significant aggregation of protein molecules covering parts of the wall surface. The rate of this protein-wall adsorption was highly sensitive to both flow rate and protein concentration. The question is whether research results with these capillaries is scalable to that in the brain. However, we currently do not know what ISF flow rates are within parenchyma, but they probably vary considerable in different parts of the brain.

In the brain parenchyma, ISF flow is often restricted by numerous large molecules projecting from the membrane wall into the ISF ECS flow path $[14,15]$. Such an obstructed path can generate both laminar and extensional shear, depending on molecular spacing, flow rate, and path geometry. Extensional shear can be simulated in laboratory experiments by sudden or gradual decreases in the inner diameter of the capillary tubing during forward flow, causing differential flow rates as the molecules flow through increasingly restricted space, resulting in a stretching of macromolecules as they flow through the narrowing capillary zone(s). If these extensionally-sheared $\mathrm{A} \beta *$ molecules then are allowed to flow into a wider 
capillary region, they experience a shear field relaxation that is the exact opposite to extensional shear, resulting in a molecular conformational change to a more relaxed, lower shear-energy state. But this relaxation to lower energy levels takes a certain amount of time to occur because of simple restrictions in intramolecular movements due to bond entanglement, solvent reorganization, etc. In the brain, as the $\mathrm{A} \beta$ molecule moves through the various brain regions that have oscillations in flow path dimensions, that molecule will also experience oscillating shear fields during this passage.

Given the complexities of the effects of shear, what is an appropriate strategic path for approaching the experimental modeling of brain shear and analysis of the products that arise from such shear processes? Ways in which some of the tentative hypotheses listed above and discussed in reference [1] can be studied experimentally are listed below and in Supplement D. First, overall goals of the general types of experiments are suggested.

\section{PROPOSED GOALS FOR SHEAR-RELATED EXPERIMENTAL AMYLOID RESEARCH}

Experimental studies of in vivo CSF and ISF flow are in their early stages. Complex flow patterns, including possible back-and-forth oscillations, have been proposed $[10,11]$. As these studies progress, the author believes that more simple, fundamental studies of laminar and extensional shear in amyloid systems should be carried out. Three overarching goals proposed are:

\section{Identify and characterize the shear-induced A $\beta$ monomer states $(A \beta *)$.}

The first and the most important of these goals is to identify and characterize the often-invoked, but thus far unidentified "misfolded" A $\beta$ intermediate [3] that is assumed to initiate a prion-like amyloid chemical cascade reaction with a precursor "seed" that initiates reactions yielding toxic oligomers and ultimately $\mathrm{AD}$ plaque. The capillary approach suggested below is an experimental method proposed for characterizing the physical and chemical properties of $A \beta^{*}$, the shear-induced, $A \beta$ intermediate proposed [1] as a potential candidate for being the shear-induced, in some cases higher energy equivalent of the quiescent "misfolded" A $\beta$ intermediate.

\section{Study the chemical reaction kinetics and identify the unstable products of the $A \beta^{*}$ intermediate.}

It is desirable to find methods for studying the chemical reactions and chemical products resulting from the reaction of $A \beta^{*}$ with other $A \beta^{*}$ and $A \beta$ molecules and with surrounding brain biochemical substances as soon as possible after $A \beta^{*}$ formation, without extensive handling and preparation for analysis.

\section{Separate, identify, and characterize unstable oligomers formed by $A \beta^{*}$ isoforms.}

Because of the instability of many of the $A \beta$ oligomers, and the great shear sensitivity of the important $\mathrm{A} \beta_{42} *$ biomarker, it is important to develop analytical methods and shear-free handling techniques for the $A \beta$ isoforms and their oligomers and fibrils, which have also been shown to be sensitive to shear [20].

\section{JUSTIFICATION FOR USING THE CAPILLARY AS A BRAIN MODEL SYSTEM}

The simplest method of generating liquid shear has been to expose sample solutions to a spinning stirring bar. However, this is not a very reproducible method, it may introduce an air-water interface that is not present in the brain, and it does not model well the highly-constricted brain liquid dynamics [14]. Much more carefully controlled quantitative shear measurements of amyloids have been made using a Couette rheometer $[4,5]$ that uses a rotating inner cylinder contained inside a stationary outside cylinder with a liquid film between the two cylinders. There have been recently reported [21] issues that may complicate the interpretations of data obtained using this method.

In this paper, an experimental method for studying liquid shear is described that generates reproducible, highly variable, and more constricted brain-like shear conditions and thus allows the testing of a number of the proposals made in the first paper in this series [1] regarding shear effects on amyloids. This technique allows spectroscopic studies of the earliest intermediates in a shear-induced amyloid cascade in which shear rates can be varied over a very wide range. In addition, by increasing the $A \beta$ concentration, this proposed system offers the opportunity for 
undertaking kinetic studies of oligomer formation from freshly-created, shear-induced $A \beta *$ intermediates with each other and with non-sheared or less-sheared $\mathrm{A} \beta$ molecules.

The current paper and one to follow in this series (Trumbore et al., unpublished experiments) describe possible experiments in which the capillary system used in our previous investigations [22-24] can be modified and usefully applied in amyloid research to investigate the role of shear on the production of various amyloid oligomer entities.

It is proposed that this capillary system offers an important analytical tool for many different aspects of amyloid-shear research, with the potential ability to follow the entire sequence of shear-induced amyloid cascade reactions leading from $A \beta$ to amyloid fibrils within one simple system, possibly in a single experiment. It is also believed that this system can be explored for use in a clinical setting to monitor changes in CSF amyloid composition. It can also test the author's hypothesis that there may be serious analytical errors in the spinal tap procedure introduced because of shear within the inside surface of the tap needle bore, which has roughly the same inner diameter and flow rates as that of the capillary used in our previous protein wall aggregation studies [18]. Depending on the outcome of the laboratory experiments suggested in this and subsequent papers, it may be possible that information that can be obtained with this system can be correlated with the disease stages in $\mathrm{AD}$ and other amyloid diseases and that the system can be used in clinical studies.

\section{CAPILLARY SYSTEM OVERVIEW}

This method inserts a short liquid cylindrical sample plug into a liquid mobile phase flowing through an otherwise empty, very small internal diameter $(\sim 0.25 \mathrm{~mm})$ stainless-steel capillary tube under bullet-shaped profile laminar flow conditions. Chromatograms are recorded by monitoring solute concentration of solutes in capillary effluent as a function of time following sample injection. Under conditions in which there is laminar flow and limited protein diffusion, chromatograms monitoring the optical absorbance of protein effluent at the exit end of the capillary yield either one or two peaks, or a peak with a shoulder, depending on the extent of solute diffusion during the passage through the capillary, for injected samples containing a single protein. In two-peak or peak plus shoulder chro- matograms, the first peak contains sample molecules that have travelled exclusively within the central part of the capillary and have been subjected to relatively small amounts of shear. The second peak or shoulder contains sample molecules that have been subjected to significant shear at or near the capillary wall. In our previous research, losses of protein molecules from the sample stream from this second peak were attributed to shear-stressed protein being adsorbed to the capillary walls [18]. The original experiments with this technique were exploratory in nature and were designed to broadly investigate the potential losses of many different types of proteins in high performance liquid chromatography capillary connecting tubing during liquid chromatography of proteins. A variety of proteins were investigated, but, unfortunately, there were no classic amyloids investigated in this early research. Although there could be a problem with metals from the stainless-steel capillary tube interacting with $A \beta$, other capillary construction materials explored such as quartz and Teflon ${ }^{\mathrm{R}}$ caused significantly more extensive and rapid protein clogging in these experiments.

A recent communication with an amyloid researcher (private communication with David Teplow) revealed that many other researchers using HPLC in their amyloid work report that there are significant losses of the amyloid protein within the HPLC system and that these researchers could not identify the location within the HPLC system of the missing amounts of amyloid sample. Given our extensive research with proteins in HPLC capillary tubing [18], it is suggested that these losses are to the walls of the small-bore connecting tubing in the system, whose inner diameter is deliberately kept very small to prevent loss of chromatographic resolution in HPLC experiments. The more important point for this paper is that it is believed that these HPLC losses originate from strong shear forces within the capillary itself. This is exactly what would be expected from a highly shear sensitive amyloid such as $\mathrm{A} \beta$.

In our limited, unpublished experiments (Trumbore, Vachet, and Say), repeat injections of amyloid samples into capillaries caused the same type of wall adsorption results as obtained in our published experiments with other types of protein. After shutting down the flow, pausing for a period of time, then starting flow again, "ghost peaks" similar to those observed with other proteins that aggregated on the wall were observed, indicating wall adsorption under shear and desorption upon release of wall shear. While none of 
these results were repeatable, they clearly indicated complex amyloid wall adsorption processes.

With further development and refinement, it is suggested that this capillary technique, under sample diffusion conditions that generate either one very sharp exponentially decaying peak (indicating severely limited solute diffusion) or two peaks or a peak and a shoulder from the injection of one sample component (indicating limited solute diffusion) may offer a promising research tool for studying the effects of shear stress on amyloids in research laboratory studies as well as other amyloid-containing biological fluids in a clinical setting, e.g., studies on extracted CSF samples. The following sections reveal in detail the origins of the double peaks and shoulders that arise from limited solute diffusion and results indicating shear-induced losses of proteins to the capillary walls.

\section{A CAPILLARY SYSTEM BASED ON LIMITED DIFFUSION: THE DETAILS}

We have previously reported [18] unusual protein behavior when aqueous cylindrical plug protein solution samples are injected into an aqueous mobile phase flowing through a stainless-steel capillary tube with a very small internal diameter. The experimental conditions used cause some of these protein molecules to undergo significant shear forces on the order of $300 \mathrm{~s}^{-1}$. However, the experimental results are not intuitive to most scientists. Thus, in our Supplementary Material, we go into some detail regarding the fluid dynamics behind these experiments, including links to animations illustrating these dynamics, links that we urge readers to explore. Below is an introduction to the more basic principles behind the experiments.

When mobile phase laminar flow starts in the capillary, the narrow capillary diameter constriction distorts an injected cylindrical sample plug to form a sample-filled, bullet- shaped downstream front profile (right side of upper figure displayed at the right) and a bullet-shaped, liquid mobile phase-filled, upstream region of the sample plug (left side of upper figure). A crude visual model for the further development of the flowing capillary plug, with no sample diffusion, is a very large number of short, very thin, constant thickness, hollow cylindrical tubes of increasing inner diameters surrounding a short, very small, solid cylinder distorted with a rounded front tip and hollow rear extending rapidly to the right with time in a telescopic manner (Fastest movement to the right in the lower figure, with no

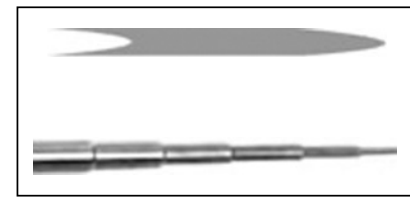
movement of the outermost cylinder on the extreme left).

This sample plug distortion is driven by the parabolic laminar flow rate profile (Fig. 1), with a zero-flow rate at the wall, where the highest shear forces are found, and with a maximum flow rate in the very center of the capillary, as indicated by the maximum velocity of the tip of the nose of the sample bullet, where the shear force is near zero because of the comparatively flat parabolic curvature of the flow profile at the extreme right of that profile shown in Fig. 1 on the right between (a) and (b). Figs. 1 and 2 represent axial cross sections along the centerline of the capillary tubes containing samples.

As the tip of the bullet nose reaches the ultraviolet light detector, the sample abruptly absorbs the UV light. The absorbance of the sample in the absorbance versus time plot in the inset of Fig. 2 starts to rise dramatically, and then falls off exponentially. The labeled specific times in Fig. 2 and its inset are: $\mathrm{A}=$ zero time with a cylindrical sample plug inserted into the solvent flow through an injection valve; $\mathrm{B}=$ bullet nose is $\sim 1 / 3$ of the way to the detector; $\mathrm{C}=$ bullet nose has just passed the detector; $\mathrm{D}=$ bullet nose is well past the detector; absorbance is due to the thin trailing sample tail.

The above discussion and illustrations have assumed that there was no solute molecule diffusion outside the borders of the gray regions (Figs. 1 and 2) representing the developing profile of the laminar flow distorted sample plug. However, such an assumption is invalid in all real cases. This interaction of plug solute molecular diffusion and distortion by laminar flow has been treated theoretically by using a number of different numerical approximation methods [25-29], including some recent calculations that will be reported in a future paper in this series (Trumbore et al., unpublished experiments).

The dynamic molecular distribution of the solute within this capillary flow system depends on the solute diffusion coefficient, the capillary diameter, the mobile phase flow rate, and the capillary length. The resulting chromatographic time versus concentration profile generated by the sample concentration detector placed at the capillary exit depends on the amount of time that the sample solute has had to diffuse during the sample plug transit. If there is a limited amount 


\section{Cylindrical Sample Plug Undergoing Bullet Flow in a Capillary Tube}

(Cross Sectional View)

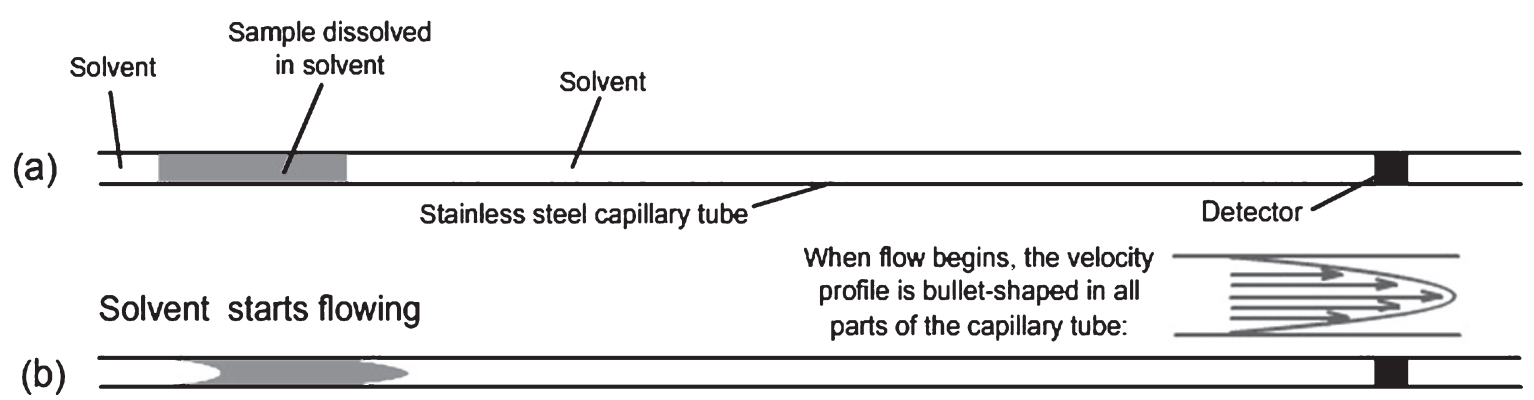

Fig. 1. Sketches of the axial cross section of the capillary tubing and injected sample.

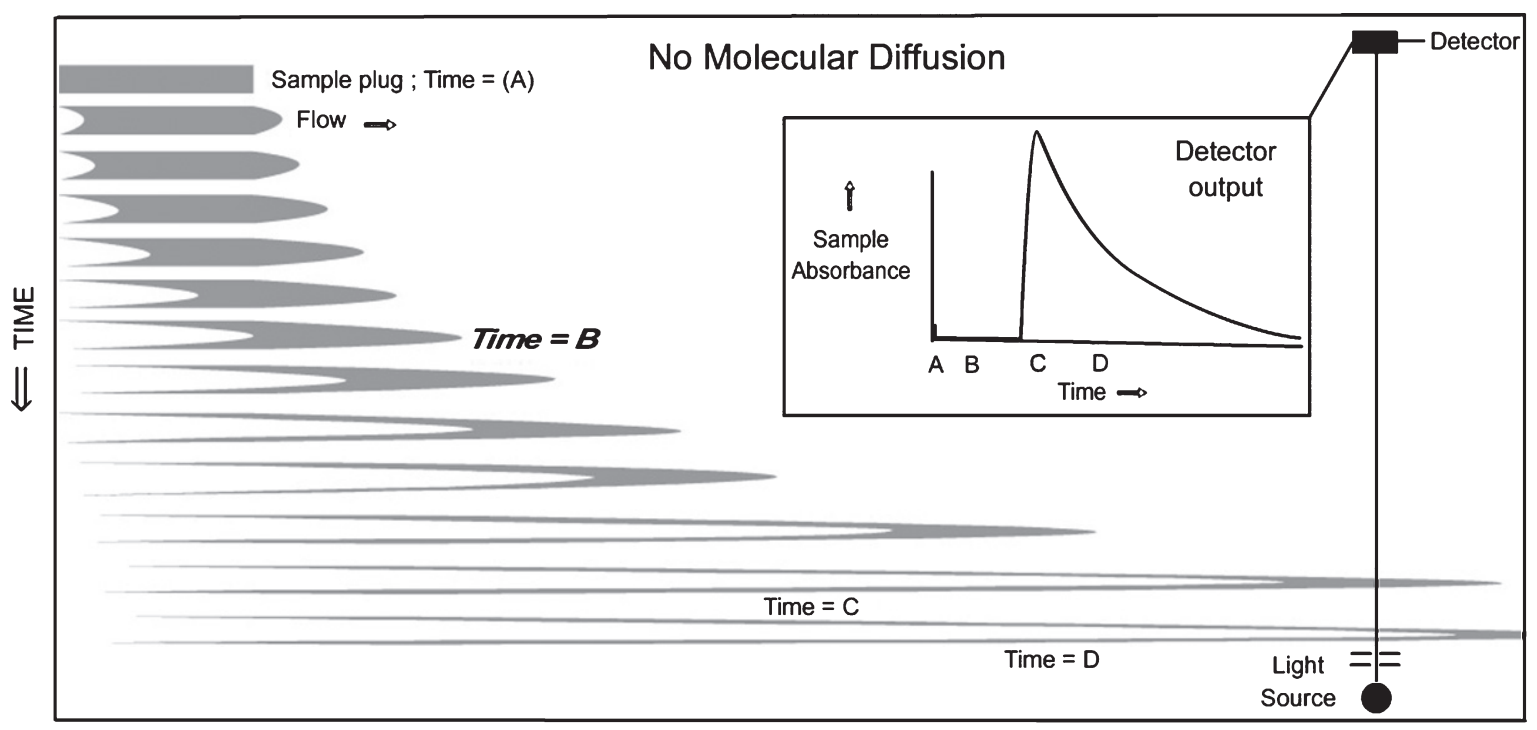

DISTANCE ALONG CAPILLARY $\Longrightarrow$

Fig. 2. Time-lapse snapshots of an axial cross section of a sample profile travelling down a capillary, distorted by laminar flow. In this figure, it is assumed that there is no diffusion of the sample molecules outside the gray borders.

of radial diffusion, there can be a significant deviation from an expected Gaussian chromatographic peak for a single component shown in Fig. 3, graph d. Instead, there is a range of single and double peaks or peaks with a prominent shoulder as shown in Fig. 3 (chromatographic profiles $a, b$, and c).

The first peak in a double peak (profile c or a sharp peak with a following shoulder, profile b in Fig. 3) represents those solute molecules that did not have a chance to diffuse very far away from the flowing sample plug segment near the center of the capillary. The second peak (right-hand shoulder in chromatogram $\mathrm{b}$ and the right-hand peak in chromatogram c, both in Fig. 3) represents those molecules that originated near or have migrated from the capillary wall and have undergone up to at least an order of magnitude higher shear force than those in the earlier sharp first peak ( $a$ and $b$ ) or shoulder on the left (c). The bottom two horizontal lines of text in Fig. 3 to the right of the (A) and (B) designations represents the wide range of experimental conditions under which one might expect to observe the type of peaks shown immediately above that text. The first line of text immediately below graphs a through d indicates the wide range of amounts of molecular diffusion needed to produce the types of graphs immediately above that text. The fluid dynamics involved in the diffusion processes that lead to these complex results in Fig. 3 are explored in more detail in the Supplement A of the Supplemental Material. The animations in Supplement A are a significant 
Typical Detector Responses for Different Amounts of Diffusion During the Sample Passage Through the Capillary

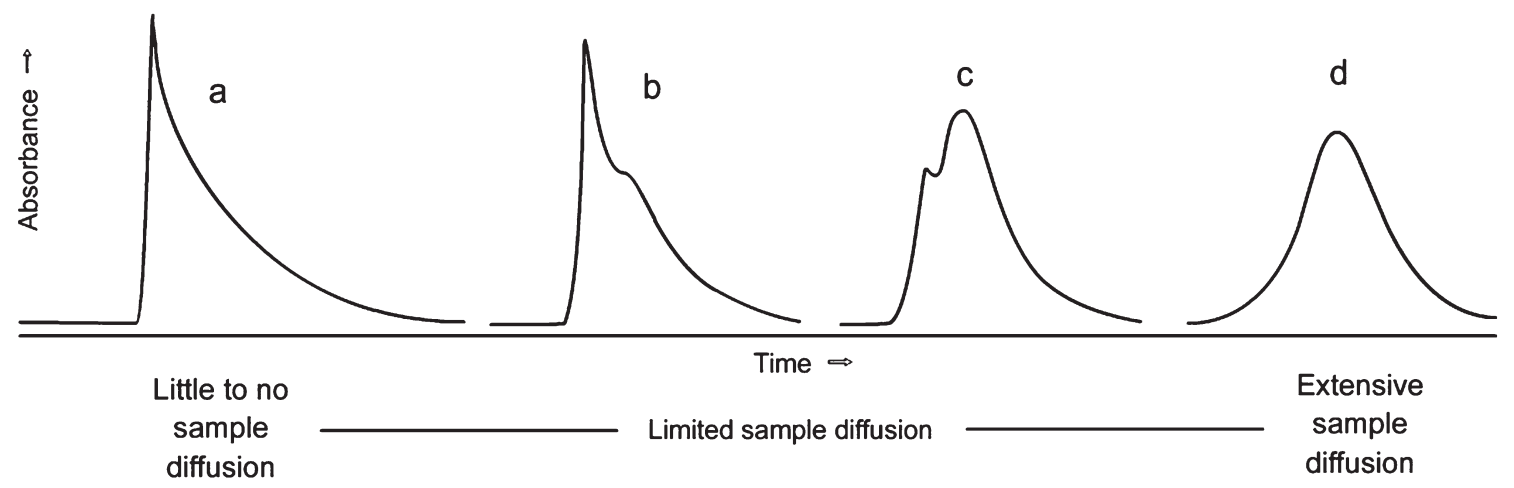

Fast sample transit through capillary
Injection of the same volume of the same sample solution in the same clean capillary
Slow sample transit through capillary
High molecular
weight sample
Injection of the same volumes of different sample solutions in the same clean capillary
Very low molecular weight sample

Fig. 3. Eluting sample concentration versus time curves under different experimental circumstances: (A) effects of different mobile phase flow rates on resulting curve shapes for a single protein sample; (B) effects of sample molecule molecular weights reflected by different diffusion coefficients.

help in visualizing the processes described above in this system.

\section{PREVIOUS CAPILLARY SYSTEM PROTEIN RESULTS}

When sample proteins of widely different molecular weights are injected into the capillary system described above at a constant flow rate, as shown in Fig. 3(B) caption conditions, the resulting chromatographic peak shapes under the same experimental conditions produced by cylindrical plug sample solution injections of these different molecules differ greatly. Similar results are obtained when samples of only one protein are injected and subjected to vastly different flow rates, as indicated in Fig. 3(A) caption conditions.

Merely determining the ratio of peak heights found in the limited sample diffusion cases shown in Fig. 3 "b" and "c" have been reported by our group to be a method for a rapid determination of protein molecular masses by comparing this ratio with a standard curve of peak height ratios versus protein molecular masses for a given flow rate [22, 23]. By altering the flow rates with a single protein sample, the shear rates increase with increasing mobile phase flow rates.
Shear forces encountered with this technique have profound effects, altering some peak shapes drastically, yielding peak shapes that are not represented in Fig. 3, especially at molecular masses above 15,000 Daltons.

Thus, these limited-diffusion peaks and shoulder regions offer a diagnostic tool for studying shearinduced protein aggregation and deposition on the capillary wall, since the right-hand second peak or shoulder is rich with molecules diffusing toward the capillary center from the wall region where there is the greatest shear, whereas the first peak represents sample that has had little to no shear and no opportunity for interaction with the wall. Elimination or losses of the second peak when there should be one indicates that sample near or at the wall, exposed to maximum shear, is unable to diffuse and migrate back toward the center and be flushed out of the capillary. This loss of sample could be caused by one of two events. The protein could be aggregated and drastically increase its molecular mass, thus reducing its diffusion coefficient, or it could be adsorbed directly on the capillary wall.

We examine in Fig. 4 an extreme example of what happens when a very high molecular weight protein is injected multiple times into the above capillary system. With very large proteins, such as fer- 


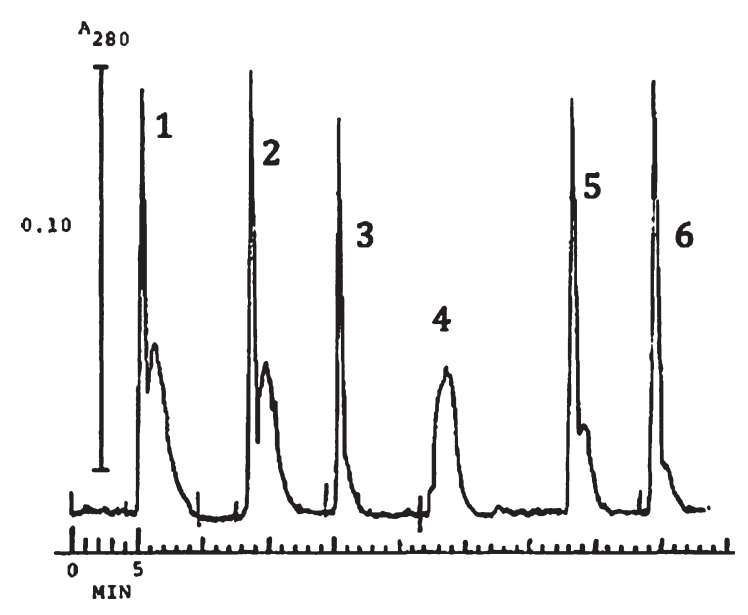

Fig. 4. Six successive injections into a $72 \mathrm{~cm} \times 0.25 \mathrm{~mm}$ I.D., 316 stainless-steel capillary; mobile phase water; flow rate $68 \mu \mathrm{1} / \mathrm{min}$; UV detector monitoring at $280 \mathrm{~nm} ; 5 \mu \mathrm{L}$ injections of a $0.13 \mathrm{mg} / \mathrm{ml}$ ferritin solution in water. No flushing or cleaning between injections. Permission granted the Journal of Chromatography [18].

ritin (molecular mass $\sim 450,000$ ), we obtained quite irreproducible results when injecting a series of subsequent identical volume samples, one injection after the other into the same capillary, with no capillary wall cleaning between injections. We see in Fig. 4 that in the first three successive injections (peak numbers $1,2,3)$, there is a gradual loss of the second peak, followed by a nearly complete loss of the first peak (injection 4), followed by a series of two injections (injection 5 and 6) in which the first peak is regained at nearly its initial height, but with very weak second peaks. We interpret this as a continual loss of protein to the walls to the point that the laminar flow was distorted completely in the fourth injection, then a partial clearing of that flow obstruction, and further wall buildup. This interpretation was confirmed based on electron micrographs of the capillary walls showing protein films and large aggregates (Supplementary Figure 5 and [18]). These results in Fig. 4 were an extreme case of many irreproducible injection studies.

Similar experiments under the same conditions as in Fig. 4 were conducted with a significantly lower molecular mass protein, e.g., $\gamma$-globulin, but with higher $\gamma$-globulin concentration injections. Under these experimental conditions and lower molecular mass there should have been two clear peaks resulting from a single injection. Hints of a slight shoulder following an initial spike did appear. Again, with multiple injections, the subsequent peaks degraded in height. The seventh peak went off scale, with sub- sequent peaks continuing the degradation in peak height of the first six injections. We designated the off-scale, seventh injection profile, with only slight tongue-in-cheek attribution, our "chromatographic heart attack." We believe these behaviors are caused by a shear-induced buildup of proteins on the capillary wall, followed by the release of one or more large particles from the wall resurrecting a more normal laminar flow pattern. Additional examples of protein losses of the types described above and in other experimental arrangements can be found in our original capillary paper [18].

A brief, high-resolution electron micrograph study was carried out on the complex pattern of the high molecular weight protein coating on the capillary walls. The protein-coating pattern demonstrates an interesting feature when there are pits in the capillary wall that appear to confirm the behavior of sheared proteins that suddenly pass near a low shear region, apparently confirming the results of Metzner et al. [30]. These results appear to demonstrate that shearstressed proteins preferentially migrate to lower shear regions into the pits when approaching zero shear locations. Discussions regarding and illustrations of these results are found in Supplement B.

\section{PROTEIN AGGREGATION AND WALL ADSORPTION UNDER SHEAR CONDITIONS}

Under our laminar flow experimental conditions, the shear rate is at a maximum next to the capillary wall, meaning the potential for protein shear-induced conformation distortion is greatest next to the wall. In our early paper [18], we suggested an explanation for the unexpected experimental results shown in Fig. 4 that is in line with suggestions made in the first paper of this series [1] and in other recent shear literature $[4,6,31]$. Briefly, these proposals are that liquid shear is responsible for altering the protein molecular conformation, thus exposing segments of the molecule that are normally shielded from an aqueous solvent environment. The protein with this unstable shear-induced conformation then undergoes molecular non-covalent-bond condensation reactions that, in the case of $A \beta$, initiate an $A \beta$ amyloid cascade reaction, forming first oligomers, then protofibrils, and finally fibrils.

A maximally stressed amyloid monomer molecule immediately next to a parenchymal membrane wall can be attracted to this wall and remain adsorbed 
because of hydrophobic and electrostatic bonding forces in a stressed conformation as long as the flow persists. Such a wall-adsorbed, stressed molecule could be an attractive adsorption site for the formation of a dimer and subsequent higher-order oligomers where each molecule is adsorbed to every other molecule in its stressed conformation. These bound oligomers, still under shear forces, could well be metastable and dissociate whenever the shear rate is reduced, such as in an oscillating back-and-forth flow that has been proposed. See Supplement C for additional discussion of protein molecular energy changes and stability considerations that lead to protein aggregation.

\section{EXPERIMENTAL CHALLENGES IN MODELING SHEAR IN BRAIN PARENCHYMA}

Using shaking, stirring, and Couette flow to provide shear forces does not appear appropriate to this author to experimentally model the shear environment experienced by A $\beta$-containing CSF or ISF in the human brain. A modified capillary system similar to that employed in our earlier research [18] would appear to better model restricted brain fluid flow within the brain vascular system and parenchyma. The diameter of our capillaries is much larger than that of the widths of the brain parenchymal ECS in which ISF flows [14]. However, the flow rates in our capillaries are also much larger than those roughly estimated to exist within the ECS, thus providing higher shear rates. The resulting shear rates in the ECS and perivascular flow channels may all be brought to within the same orders of magnitude with the appropriate adjustments in capillary diameter and mobile phase flow rates once the in vivo rates have been reported. Shear rates in a capillary are inversely proportional to the cube of the capillary radius, thus making the shear rate exceedingly sensitive to brain flow path width parameters as they narrow to very small values. In other words, even though ISF parenchymal flow rates may slow down by many orders of magnitude by narrowing the width parameter, this same parameter also increases the shear rates by at least many orders of magnitudes.

$\mathrm{A} \beta$-containing ISF must flow through a tortuous path between neurons, astrocytes, and other glial membranes as well as weaving their way through molecules jutting out from their membrane anchors and other molecular flow inhibitors in the perineuronal networks [15]. When a commercial polymer solution is forced to flow through a wire mesh, there are circumstances [30] where some synthetic polymers produce a stringy insoluble polymer on the downstream side of the mesh. The theory put forth to explain this effect by Metzner et al. [30] is that shearstressed polymers migrate toward lower shear stress regions, in this case immediately behind the downstream sides of the flow-impeding wires in the wire mesh. These molecules also become shear-oriented as they pass around the flow-resisting wires, so, as they migrate preferentially toward the region behind the wires, they probably aggregate in an oriented manner, building up solids that can further impede liquid flow. Those molecules closest to the wall and therefore subjected to high laminar shear can be attracted to the wall provided there are attractive molecular forces between the wall and the stretched polymer. Such attraction is certainly likely in the case of an in vivo membrane with a perineuronal protein complex anchored in the membrane and a shear-stressed amyloid protein in contact with that protein.

IDPs, such as $A \beta$, are probably more subject to shear-induced conformational change than other proteins that maintain a single, more tightly bonded, native conformation in the absence of shear. Shear, such as that generated by Couette flow, can induce conformational changes in amyloid molecules that initiate a chain of reactions leading to the formation of amyloid fibrils [4, 31]. Stirring of similar solutions also accelerates the formation of amyloid fibrils, especially $A \beta_{42}$ [20]. However, on a molecular scale, this stirring corresponds to flow around a single object, not between two objects.

The inside diameter of capillaries used in our studies $(\sim 250 \mu \mathrm{m})$ is larger than that estimated for the openings existing within the perivascular system $(\sim 5-10 \mu \mathrm{m})$ and especially larger than the estimated flow paths among the astrocytes and neurons in the parenchyma $(\sim 50 \mathrm{~nm})$ [14]. The average shear rates in our studies $\left(\sim 300 \mathrm{~s}^{-1}\right)$ may be of the same order of magnitude of those experienced by $\mathrm{A} \beta$-containing CSF in the vascular parts of the brain because of our faster flow rates. The flow rates of ISF in the parenchyma are unknown. However, there is some evidence for a slow flow of tracers through the parenchyma of a mouse [32], but these flow rates are still many orders of magnitude slower than those in our capillary experiments. However, there is at least a $\sim 5,000$-fold difference in flow restrictor dimensions 
in our capillaries and the extra cellular dimensions of the parenchyma channels [14]. Thus, very slow ISF flow rates in the very small, highly congested parts of the brain parenchyma may very well generate shear conditions for ISF fluid perhaps an order of magnitude lower than those of CSF in the vascular system. Flow rates in our capillary experiments were steady, whereas flow rates of CSF through the brain parenchyma may be both pulsatory and oscillatory $[10,11]$, possibly giving the shear-stressed protein molecules a chance to relax to their unstressed or possibly to a less-stressed conformation. This relaxation lifetime of the shear-stressed molecule determines the probability that a shear-stressed molecule will meet and combine with another nearby shear-stressed molecule, a probability that is non-linearly dependent on $A \beta$ concentration. If the concentration of $A \beta$ increases for some reason, e.g., because of $\mathrm{AD}$, this can significantly accelerate $A \beta$ oligomer and fibril formation.

\section{EFFECTS OF NATURAL SHEAR RELAXATION PERIODS DURING SLEEP}

It is reported that the ECS between neurons and astrocytes is larger during the sleeping period for mice than in their awake period [8]. If the changes in ISF channel dimensions proposed by Iliff and coworkers [8] for sleeping mice can be extrapolated to humans, then sleep could bring about drastic reductions in shear rates experienced by $\mathrm{A} \beta$ molecules dissolved in ISF and those adsorbed on membrane walls and in the perineuronal network.

However, not only would shear relaxation increase the proposed clearance of $A \beta$ from the brain parenchymal fluids, it would also allow shearinduced metastable aggregates, including those adsorbed on the membrane, to be exposed to lower shear rates. This lower shear sleep regime could well enhance the dissolution of those surface $A \beta$ aggregates whose aggregated wall structure depends on the presence of a high-shear environment. Our recent, limited capillary experiments (Trumbore, Vachet, and Say, unpublished experiments) with $A \beta$ under experimental shear conditions have demonstrated that shear-induced amyloid capillary coatings at least partially dissolve back into the mobile phase when mobile phase flow is halted. Also, in any of the proposed back-and-forth oscillations of either ISF or CSF $[10,11]$, there would be minima in the shear rates in these oscillations where dissolution of the aggregates could possibly occur. This net dissolution rate would depend upon both the relaxation lifetime of the dissolution process under minimum shear and the oscillation frequency. Both of these processes can be studied experimentally under controlled conditions in the proposed capillary system.

Pulsatory and oscillatory forcing of the CSF and ISF through many brain regions provides numerous opportunities for shear-induced amyloid molecule distortion, extension, and subsequent aggregation [1]. Amyloid aggregation is known to be amyloid concentration dependent in both quiescent and sheared solutions. Anything that prevents flushing of $A \beta$ out of brain parenchyma, thereby increasing the $A \beta$ concentration, will tend to increase the likelihood of amyloid oligomer formation and to maintain its stability if this formation is a reversible reaction. That is, higher $\mathrm{A} \beta$ concentrations shift cascade product equilibria toward the higher order $A \beta$ complexes such as oligomers and protofibrils.

Given the above background about our capillary system, recent experimental observations on brain fluid flow, and speculations regarding the role of shear in amyloid research, what are the types of experiments that can be carried out using our capillary system?

\section{PROPOSED CAPILLARY SYSTEM EXPERIMENTS FOR BASIC SHEAR RESEARCH}

It is proposed that our modified capillary column system can be used productively to test in Supplement D many of the numerous speculations made above, and in the previous paper [1]. This capillary system provides a very wide range of known, reproducible shear rates, ranging from near zero in the center of the capillary to maximum at the capillary wall. These shear rates are easily varied over a very wide range by changing either the flow rate or capillary dimensions or both. The system has been shown to be able to provide significant shear-induced phenomena in very short time periods. It is believed that this capillary system can, with appropriate alterations and variations in design, provide a model system for the study of the complex flow and possible pulsatory [33] and oscillatory [10, 11] shear stress patterns within the brain that have been recently proposed. Also, given the experimental results of Metzner et al. 
[30] demonstrating stress-induced formation of precipitates of synthetic polymers flowing through a flow-constricting grid, it is believed that qualitatively similar shear flow patterns can be formed in the capillary system. These would be studied by properly manipulating flow rates and using appropriate combinations of capillary sizes and flow impediments within the capillaries to produce extensional as well as laminar shear patterns that simulate those present in the brain.

The exploratory results of our previous papers using capillaries in protein studies were obtained in the early 1980s, with laboratory equipment that was far less than optimal. Because the retired author of this paper is limited by lack of access to laboratory facilities, he has been unable to carry out the large number of obvious experiments that logically flow from the above and previous paper [1] discussions. He therefore strongly urges scientists who are actively engaged in amyloid research to consider carrying out one or more of the suggested experiments in Supplement D with their own amyloid systems. Experiments proposed in Supplement D use $A \beta$ as the amyloid molecule but could also use prion proteins or other amyloid monomers. Listed in Supplement D are some of the more urgent experiments proposed by the author. There are many more experiments that the reader may imagine that could be designed for their own amyloid system.

\section{POTENTIAL SERIOUS SHEAR-RELATED ERRORS IN SPINAL TAP ANALYTICAL RESULTS}

The needle commonly used for spinal tap has approximately the same inner diameter as that used in our early capillary experiments described above [18]. The spinal tap method allows spinal CSF to flow through this needle without suction and allows the CSF to freely drip into collection tubes without any back pressure. However, some laboratories do apply suction to accelerate this flow and some use smaller needles ([34] and private communication with Anne Fagan). Both of these procedures appear to give rise to flow rates that exceed those in our studies with proteins and $A \beta$, thus exceeding the shear rates experienced by dissolved proteins and $A \beta$ in our capillary experiments [18] where shear-induced aggregation of many different proteins occurred, producing adsorbed protein deposits on the inner walls of the capillary.
Given this discovery, the reports of problems with HPLC A $\beta$ analyses, and our more recent preliminary results with $A \beta$ (Trumbore, Vachet, and Say, unpublished experiments), the serious concern is raised here that CSF flowing directly from the spinal column and that is exposed to shearing next to the spinal tap needle inner walls that is sufficient to induce $A \beta$ aggregation and adsorption on this inside surface of the needle and also possibly to induce oligomer formation in the fluid portion of the spinal tap needle near the wall can cause serious errors in all analyses of the CSF amyloid content. This would be especially true if suction is used to increase the CSF flow rate out of the spinal CSF reservoir. It is concluded by this author that experiments with various CSF flow rates through these same spinal tap needles are urgently needed to determine whether these predicted losses of amyloids to the inside walls of the needles are occurring during CSF collection. Such studies should make use of the multiple successive injection, limited diffusion, double peak behavior that we have used to study wall adsorption described above and in [18].

It has been proposed that the spinal fluid flow is oscillating back and forth $[10,11]$ with a net forward flow. This could cause maxima in the shear stress values at the peaks of the oscillations. It has also been proposed that in the aged patient with increasing atherosclerosis, spikes in blood pressure-and possibly also in CSF flow- and shear-rates-become much stronger. The question that arises is, are these two factors increasing pulsed CSF flow rates during the spinal tap process, resulting in increased shear-induced aggregation to form oligomers in solution and amyloid wall adsorption inside the needle.

As a result, in the opinion of this author, the first priority should be to explore this potential problem in a clinical setting. For example, patient experiments should be conducted in which various amounts of back pressure is applied to slow down and stabilize the spinal tap flow rate and eliminate any possible back-and-forth or strong pulse fluctuations of CSF shear rate next to the needle surface and to determine whether this alters analytical CSF data when compared with similar data obtained from several CSF samples from the same patient during the same time period, one in which the CSF flows freely through the needle, the other in which syringe suction is applied to obtain the sample. This author believes that top priority should be given to these proposed clinical experiments, since CSF analyses are used as 
a prime biomarker for $\mathrm{AD}$. Critical measurements in laboratory and clinical experiments should be able to determine the effects of back pressure and CSF flow rate on the $A \beta_{42} / A \beta_{40}$ ratio, especially given the greater sensitivity of the $A \beta_{42}$ isoform to shear than that of $A \beta_{40}$. It is anticipated that this ratio would be very much dependent on the flow rate of the CSF flowing from the patient. At very low shear rates, only $A \beta_{42}$ might adsorb to the wall. At higher shear rates, both $A \beta_{40}$ and $A \beta_{42}$ probably would wall adsorb, and the ratio of these two isoforms probably depending on the flow rate.

\section{SUMMARY AND CONCLUSIONS}

Shearing of aqueous amyloid solutions has been observed to initiate a chemical reaction cascade leading from an amyloid protein monomer through oligomers to protofibrils and ultimately high molecular weight fibrils. However, there are reports that both the kinetics of formation and the structure of these fibrils are different under shear and nonshear conditions. There is increasing experimental evidence that $A \beta$-containing fluids in the brain are undergoing flow conditions that probably generate both laminar and extensional shear forces, indicating that the amyloid cascade can possibly be initiated by shear processes within the brain, especially with highly shear-sensitive $A \beta_{1-42}$ contained in the brain parenchyma. It is proposed in this paper that the capillary system described in this paper can provide a highly flexible system with which to study both shear-induced amyloid cascade and aggregation and surface-adsorption processes because of the variable shear rates that can be easily controlled and quantitatively measured in fast, relatively simple experiments. It is further suggested that this capillary system can be used to model the type of flow processes taking place as $\mathrm{A} \beta$-containing CSF flows and is pulsed through arterial channels leading into the brain as well as ISF flowing more slowly through the tangled web of neurons and glial cells within the brain parenchyma.

There are many advantages of this capillary system. Besides its simplicity, it is fast, quantitative, reproducible, relatively inexpensive, and flexible in its ability to model the various parts of the brain where shear might be important. Different designs of the constricted flow geometry are possible other than those with a circular cross section of the capillary that confine the flowing amyloid solution and generate comparatively large wall shear forces in selected portions of the flow channel. The ability to vary dimensions and flow rates allows a large range of shear rates to be studied. Different flow restriction dimensions in the same experiment allow the study of extensional shear. Different types of detectors can be used to study the fluid effluent at the mobile phase exit end of the capillary including UV, IR, Raman, light scattering, and mass spectroscopic monitoring. Stopped flow experiments can be carried out on samples immediately following being subjected to different shear rates to study spectroscopic properties and relaxation rates of shear-excited $A \beta^{*}$ molecules. Different A $\beta$ concentrations can be used to explore the kinetics of reactions between two similarly sheared amyloid molecules.

Recent in vivo research results indicate that amyloid monomers contained in CSF and ISF are continually being forced through different parts of the brain, some in a pulsating and also possibly oscillating manner. Therefore, it is suggested that amyloid research should also expose amyloid solutions to such pulsatory as well as oscillatory flows in this capillary system. It is suggested that aliquots of effluent from this capillary system be used for toxicity, seeding experiments, and qualitative or quantitative analysis to test the effects of variable shear forces within different regions of the capillary. A large number of experiments are suggested. Others will undoubtedly occur to the reader for their particular amyloid system.

This retired author and experimenter strongly urges further research into the role of shear in all aspects of amyloid diseases. This is especially important for those who analyze CSF for amyloid composition data obtained through spinal taps, since the needles used in this procedure have approximately the same diameter and even greater flow and thus larger shear rates than those that caused shear-induced protein wall coatings in our research [18]. The danger of shear-induced alteration of CSF amyloid composition is especially likely when finer needles are used, especially when suction is used to enhance the flow rate.

\section{ACKNOWLEDGMENTS}

The author acknowledges helpful discussions with Abraham Lenhoff, Herbert Blades, Jeffery Iliff, Lila Gierasch, Arthur Metzner, Richard Vachet, Dave 
Dunstan, Lorna Alston, David Colby, David Talaga, Richard Kline, Maxwell Siegel, Susan Trumbore, and Virginia Ahrens.

The author's disclosure is available online (http:// j-alz.com/manuscript-disclosures/17-0259r2).

\section{SUPPLEMENTARY MATERIAL}

The supplementary material is available in the electronic version of this article: http://dx.doi.org/ 10.3233/JAD-170259.

\section{REFERENCES}

[1] Trumbore CN (2016) Shear-induced amyloid formation in the brain. I. Potential vascular and parenchymal processes. J Alzheimers Dis 54, 457-470.

[2] Butterfield S, Hejjaoui M, Fauvet B, Awad L, Lashuel HA (2012) Chemical strategies for controlling protein folding and elucidating the molecular mechanisms of amyloid formation and toxicity. $J$ Mol Biol 421, 204-236.

[3] Knowles TPJ, Vendruscolo M, Dodson CM (2014) The amyloid state and its association with protein misfolding diseases. Nat Rev Mol Cell Biol 15, 384-397.

[4] Dunstan DE, Hamilton-Brown P, Asimakis P, Ducker W, Bertolini J (2009) Shear flow promotes amyloid- $\beta$ fibrilization. Protein Eng Des Sel 22, 741-746.

[5] Bekard IB, Asimakis P, Bertolini J, Dunstan DE (2011) The effects of shear flow on protein structure and function. Biopolymers 95, 733-745.

[6] Ashton L, Dusting J, Imomoh E, Balabani S, Blanch EW (2010) Susceptibility of different proteins to flowinduced conformational changes monitored with Raman spectroscopy. Biophys $J$ 98, 707-714.

[7] Smock RG, Gierasch LM (2009) Sending signals dynamically. Science 324, 198-203.

[8] Xie L, Kang H, Kang H, Xu Q, Chen MJ, Liao Y, Thiyagarajan M, O'Donnell J, Christensen DJ, Nicholson C, Iliff JJ, Takano T, Deane R, Nedergaard M (2013) Sleep drives metabolite clearance from the adult brain. Science 342, 373377.

[9] Weller RO, Preston SD, Subash M, Carare RO (2009) Cerebral amyloid angiopathy in the aetiology and immunotherapy of Alzheimer disease. Alzheimers Res Ther $1,6$.

[10] Brinker T, Stopa E, Morrison J, Klinge P (2014) A new look at cerebrospinal fluid circulation. Fluids Barriers CNS 11, 10.

[11] Hladky S, Barrand M (2014) Mechanism of fluid movement into, through and out of the brain: Evaluation of the evidence. Fluids Barriers CNS 11, 26.

[12] Bakker ENTP, Bacskaf BJ, Arbel-Ornath M, Aldea R, Bedussil B, Morris AWJ, Weller RO, Carare RO (2016) Lymphatic clearance of the brain: Perivascular, paravascular and significance for neurodegenerative diseases. Cell Mol Neurobiol 36, 181-194.

[13] Blennow K, Zetterberg H (2015) The past and the future of Alzheimer's disease CSF biomarkers-a journey toward validated biochemical tests covering the whole spectrum of molecular events. Front Neurosci 9, 345.
[14] Wolak, DJ, Thorne RG (2013) Diffusion of macromolecules in the brain: Implications for drug delivery. Mol Pharm 10, 1492-1504.

[15] de Winter F, Kwok JC, Fawcett JW, Vo TT, Carulli D, Verhaagen $J$ (2016) The chemorepulsive protein semaphorin $3 \mathrm{~A}$ and perineuronal net-mediated plasticity. Neural Plast 2016, 3679545.

[16] Kotler SA, Walsh P, Brender JR, Ramamoorthy A (2014) Differences between amyloid-beta aggregation in solution and on the membrane: Insights into elucidation of the mechanistic details of Alzheimer's disease. Chem Soc Rev 43, 6692-6700.

[17] Radford SE, Weissman JS (2012) Editorial overview: Special issue: The molecular and cellular mechanisms of amyloidosis. J Mol Biol 421, 139-141.

[18] Trumbore CN, Tremblay R, Penrose J, Mercer M, Kelleher F (1983) Unusual flow behavior in high-performance liquid chromatography capillary tubing. J Chromatogr 280, 43-57.

[19] Drolle E, Hane F, Lee B, Zoya Leonenko BL, Leonenko Z (2014) Atomic force microscopy to study molecular mechanisms of amyloid fibril formation and toxicity in Alzheimer's disease. Drug Metab Rev 46, 207-223.

[20] Cohen SI, Linse S, Luheshi LM, Hellstrand E, White DA, Rajah L, Otzen DE, Vendruscolo M, Dobson CM, Knowles TP (2013) Proliferation of amyloid- $\beta 42$ aggregates occurs through a secondary nucleation mechanism. Proc Natl Acad Sci U S A 110, 9758-9763.

[21] McBride SA, Sanford SP, Lopez JM, Hirsa AH (2016) Shear-induced amyloid fibrillization: The role of inertia. Soft Matter 12, 3461-3467.

[22] Trumbore CN, Grehlinger M, Stowe M, Kelleher FM (1985) Further experiments on a new, fast method for determining molecular weights of diffusing species in a liquid phase. J Chromatogr 322, 443-454.

[23] Kelleher FM, Trumbore CN (1984) Dispersion of protein solutions in short, open capillary tubes as a method for rapid molecular weight determination. Anal Biochem 137, 20-24.

[24] Trumbore CN, Jackson LM, Bennett S, Thompson A (1989) High-speed analytical sensor for in-line monitoring of dissolved analytes flowing in a tube employing a combination of limited diffusion, laminar flow, and plug solvent injections. J Chromatogr 481, 111-120.

[25] Atwood JG, Golay MJE (1981) Dispersion of peaks by short straight open tubes in liquid chromatography systems. J Chromatogr 218, 97-122.

[26] Shankar A, Lenhoff AM (1989) Dispersion in laminar flow in short tubes. AIChE J 35, 2048-2052.

[27] Shankar A, Lenhoff AM (1991) Dispersion in round tubes and its implications for extracolumn dispersion. J Chromatogr 556, 235-248.

[28] Lighthill MJ (1966) Initial development of diffusion in Poiseuille flow. IMA J Appl Math 2, 97.

[29] Vrentas, JS, Vrentas CM (1988) Dispersion in laminar tube flow at low Peclet numbers or short times. AIChE J 34, 1423.

[30] Metzner AB, Cohen Y, Rangel-Nafaile C (1979) Inhomogeneous flows of non-newtonian fluids: Generation of spatial concentration gradients. J Nonnewton Fluid Mech 5, 449462.

[31] Teoh CL, Bekard IB, Asimakis P, Griffin MDW, Ryan TM, Dunstan DE, Howlett GJ (2011) Shear flow induced changes in apolipoprotein C-II conformation and amyloid fibril formation. Biochemistry 50, 4046-4057. 
[32] Iliff JJ, Wang M, Liao Y, Plogg BA, Peng W, Gundersen GA, Benveniste H, Vates GE, Deane R, Goldman SA, Nagelhus EA, Nedergaard M (2012) A perivascular pathway facilitates CSF flow through the brain parenchyma and the clearance of interstitial solutes, including amyloid beta. Sci Transl Med 4, 147ra111.

[33] Stone J, Johnstone DM, Mitrofanis J, O'Rourke M (2015) The mechanical cause of age-related dementia (Alzheimer's
Disease): The brain is destroyed by the pulse. J Alzheimers Dis 44, 355-373.

[34] Fagan AM, Younkin LH, Morris JC, Fryer JD, Cole TG, Younkin SG, Holtzman DM. (2000) Differences in the $A \beta 40 / A \beta 42$ ratio associated with cerebrospinal fluid lipoproteins as a function of apolipoprotein E genotype. Ann Neurol 4, 201-210. 